\title{
UN ANÁLISIS DE LA SEGREGACIÓN SOCIO-ESPACIAL EN EL GRAN RESISTENCIA
}

\section{A SOCIO-SPATIAL SEGREGATION ANALYSIS IN GRAN RESISTENCIA}

\author{
Prof. Aníbal Marcelo Mignone
}

Auxiliar Docente del Departamento de Geografía

Departamento de Geografía- UNNE

\section{Resumen}

Entendiendo a la segregación socio-espacial como la concentración espacial de personas consideradas dentro de una misma categoría social, el trabajo analiza la segregación socio-espacial en el Gran Resistencia, a nivel de radio censal, y utilizando las variables provistas por el censo nacional de Población de 2001.

Se utilizan los índices que miden diferentes dimensiones de la segregación, como la igualdad, concentración y autocorrelación espacial, complementado con el uso de Sistemas de Información Geográfica (SIG).

\begin{abstract}
Understanding socio-spatial segregation and spatial concentration of people considered within the same social category, the paper examines the socio-spatial segregation in Greater Resistance, census-level radio, and using the variables provided by the national census population 2001. Indices are used to measure different dimensions of segregation, such as equality, concentration and spatial autocorrelation, supplemented by the use of Geographic Information Systems (GIS).
\end{abstract}

\section{Palabras Claves.}

Segregación socio-espacial; Gran Resistencia; Sistemas de Información Geográfica

\section{Keywords:}

Socio-spatial segregation; Gran Resistencia; Geographic Information Systems

\section{1- INTRODUCCIÓN}

Las investigaciones concuerdan que en siglo $\mathrm{XX}$, los cambios sociales comienzan a producirse con la crisis del sistema económico fordista, que en la mayor parte del mundo condujeron a una reestructuración de ciertos contextos espaciales, especialmente en las ciudades (Janoschka, 2002). Ese proceso de cambio tuvo como contraparte social, la disolución de las "clases" tradicionales y la tendencia a la formación de una amplia pluralidad de ámbitos sociales en las sociedades urbanas.

Así, se fue mutando de una ciudad con núcleos tradicionales perfectamente reconocidos, a una con mayor complejidad. Esta situación, es factible de observar en los conjuntos urbanos latinoamericanos, los cuáles han presentado profundas modificaciones en su estructura interna, especialmente en los que respecta a las grandes metrópolis y a las ciudades intermedias.

En este sentido, el aglomerado conocido como Gran Resistencia1, se considera como una ciudad de tamaño intermedio2 y ha visto aumentar rápidamente su población a partir de la década de 19603, con lo cuál ha ido complejizando su propia organización interna. A medida que se ocuparon los espacios libres en el centro, se produjo la necesidad y el impulso de ocupar nuevos terrenos hacia la periferia con función residencial (Foschiatti de Dell' Orto, 1992). Este crecimiento vertiginoso de propagación significó una desarticulación espacial, provocando falencias en la distribución de los servicios e insuficiencia en las áreas periféricas.

El crecimiento del espacio urbanizado de la capital chaqueña, a partir de la década de 1990, operó según tres mecanismos principales: el más importante de ellos fue el de los asentamientos informales (usualmente por el mecanismo de la ocupación ilegal de terrenos), acompañados por los 
barrios planificados y la construcción de edificios en torre en la zona céntrica (cf. Valenzuela de Mari, 2000). A esta situación, actualmente se suma la construcción de barrios privados, primordialmente en la periferia noreste del municipio Resistencia.

En el caso del incremento en el número de barrios informales, uno de sus principales factores fueron las políticas de liberalización del mercado de suelo urbano, que generalmente por su alto costo, permitió el acceso a un conjunto acotado de población, mientras que los grupos de menores recursos tuvieron como principal mecanismo la ocupación ilegal de los terrenos. A esto se sumó el fenómeno de la relocalización espacial de la población (Meichtry y Mignone, 2003; Mignone, 2005), primordiamente de barrios de clase media-baja y baja, que ante la imposibilidad de acceder a un terreno propio, optaron por ocupar uno de manera ilegal4.

Por otra parte, la capital de Chaco tuvo cierto crecimiento económico en la primera mitad de la década pasada, pero posteriormente pasó a depender en gran medida de las transferencias de los recursos públicos, especialmente en políticas sociales (Barreto y Sánchez, 2000). En efecto, la creación de viviendas de tipo social por parte del estado provincial, ha llevado a una gran concentración de moradas en terrenos de bajo costos y con problemas ambientales, localizados en zonas periféricas en cuyas proximidades se encuentran grupos de nivel socioeconómicos bajos.

Por lo tanto, el crecimiento urbano de la capital ha dado lugar a una vasta periferia con calidad heterogénea en lo que hace a servicios, equipamiento e infraestructura, opuesta a la denominada área central, casco central o casco histórico dónde se concentran las principales actividades (administrativas, comerciales, financieras, gubernamentales, etc.), con convergencia de los principales medios de transporte y usualmente, un fuerte crecimiento en altura. La ocupación de este sector central está asociado (entre otras causas) a una cuestión de "prestigio" o de "estatus social", lo que provoca una "competencia" para acceder a estos espacios. Esta situación da lugar a la segregación residencial de las personas y de los distintos tipos de actividades que realizan; así como el dominio de un grupo sobre el otro estará basado en el poder económico de los grupos poblacionales y en el valor del suelo urbano (Buzai, 2003:38).

Todos los factores mencionados, han generado una fragmentación espacial y han acrecentado las condiciones de segregación socioespacial, las que han adquirido una gran visibilidad y trascendencia, por considerar su asociación con un proceso de reproducción de las desigualdades socioeconómicas (Arraigada y Rodríguez, 2003) y por otra parte, porque se deriva de la concentración de núcleos socioeconómicos altos.

\section{2- CONCEPTUALIZANDO LA SEGREGACIÓN SOCIPESPACIAL}

El uso del término segregación ha generado situaciones muy ambiguas ya que se aplica a una gran diversidad de circunstancias, en algunas de las cuales no es tan clara la intencionalidad, así como que resulta más difícil distinguir a los grupos involucrados (cf. Formiga 2000). Además, la conceptualización es muy diferente según la disciplina que analiza el fenómeno y tiene el inconveniente que en el imaginario social, el uso del término segregación implica discriminación, o presupone una idea similar.

Una segregación socioespacial en el ámbito urbano, implica la distancia física entre la localización residencial de los grupos sociales, de esta manera se entiende a la segregación como la tendencia a la organización del espacio en zonas de fuerte homogeneidad social interna y de fuerte disparidad social entre ellas, entendiéndose esta disparidad no sólo en términos de diferencia, sino de jerarquía (Castells, 1974).

Según Brun (en Brunet y Rhein 1994 citado por Formiga, 2000), la aplicación más precisa y menos discutible del proceso de segregación en el ámbito urbano, corresponde a los espacios ocupados por los grupos más pobres, los que establecen una clara diferenciación en la morfología urbana. Estas áreas, generalmente localizadas en el borde de la ciudad, presentan una alta asociación de indicadores que revelan las condiciones de carencia y privación que se relacionan tanto con el grupo de población como en su hábitat, que pone de manifiesto la exclusión económica, social y cultural de estos grupos.

De esta manera, se considera a la segregación socioespacial como el grado de proximidad espacial o la aglomeración geográfica de personas de una misma categoría social (cf. Sabatini, 2000). Por lo tanto, remite a las diferencias o desigualdades sociales que existen en el espacio físico, dónde los sujetos se encuentran separados en categorías que presentan cierto grado de distinción

Publicado en formato digital: Prof. Aníbal Marcelo Mignone. UN ANÁLISIS DE LA SEGREGACIÓN SOCIOESPACIAL EN EL GRAN RESISTENCIA. Revista Geográfica Digital. IGUNNE. Facultad de Humanidades. UNNE. Año 8. No 16. Julio - Diciembre 2011. ISSN 1668-5180 Resistencia, Chaco.

En: http://hum.unne.edu.ar/revistas/geoweb/default.htm 
jerárquica o valorativa. A ello, se suma el hecho de existir una ausencia o escasez relativa de mezclas socioeconómicas dentro de las unidades territoriales de la ciudad (cf. Rodríguez Vignoli, 2001). Además, la segregación socio-espacial indirectamente estaría determinando el valor del suelo de cada área de la ciudad, según la accesibilidad de determinados grupos sociales a diferentes zonas del ámbito urbano, de acuerdo con el poder adquisitivo de los mismos.

Considerando estas definiciones, el trabajo se propone a través de una visión cuantitativa, medir la segregación socioespacial en el Gran Resistencia para el año 2001, utilizando las variables educativas y laborales provistas por el censo Nacional de Población y Viviendas de 2001. Se trabaja a nivel de radio censal y se utilizan los índices que miden diferentes dimensiones de la segregación, como la igualdad, concentración y autocorrelación espacial, complementado con el uso de Sistemas de Información Geográfica (SIG).

\section{3- LAS VARIABLES UTILIZADAS Y LOS ÍNDICES DE SEGREGACIÓN APLICADOS}

Se utilizaron los datos correspondientes al Censo Nacional de Población 2001 y se los trabajó inicialmente mediante el uso del software REDATAM+SP desarrollado por CELADE- CEPAL que permite procesar la información contenida en bases de datos comprimidas y muy extensas, como las provenientes de censos de población completos. Esto permite alcanzar el mayor nivel de desagregación geográfica, como son los radios censales.

Se ha optado por considerar las variables de población vinculados a cuestiones como la educación y el empleo, las cuáles fueron las siguientes:

- Población analfabeta de 10 y más años (Analfabetos)

- Población de 6 a 14 años con abandono escolar (Abandono escolar)

- Población que asiste a establecimiento educativo privado (Establecimiento Privado)

- Población de jefes de hogar con estudio primario incompleto (Jefe Primaria)

- Población jefes de hogar con estudios terciarios o universitarios completo (Jefe Superior)

- Patrones con calificación profesional en establecimiento de 6 o más empleados (Patrones) aporta)

- Población ocupada que no aporta para obra social ni le descuentan aportes jubilatorios (No

- Población ocupada no calificada (Baja calificación)

- Población sin cobertura de obra social o mutual (Sin obra social)

En cuanto a los indicadores de segregación, estos surgen de la clasificación hecha por Massey y Denton (1988), donde los autores proponen que la segregación se manifiesta a través de varias dimensiones como: igualdad, concentración y agrupamiento. Para cada dimensión existe al menos un indicador básico de medida y a través de su aplicación empírica se demuestra estadísticamente que las medidas obtenidas por los diferentes indicadores son independientes entre sí (Lavía Martínez, 2008).

Para el análisis, se han utilizado los siguientes índices:

\begin{tabular}{|l|l|}
\hline * Índices de igualdad & $\begin{array}{l}\text { - Índice de Segregación (IS) } \\
\text { - Índice de Disimilitud de Duncan (ID) }\end{array}$ \\
\hline * Índices de concentración & - Índice Delta (DEL) \\
\hline * Índices de autocorrelación & $\begin{array}{l}\text { - Índice de Morán Global (I) } \\
\text { - Índice de Morán Local ( } \mathrm{I}_{\mathrm{i}} \text { ) }\end{array}$ \\
\hline
\end{tabular}

\section{4- LOS ÍNDICES DE IGUALDAD}

En el estudio, se utilizaron los índice de Segregación (IS) y de Disimilaridad de Duncan (ID), cuyos valores varían entre cero y uno, y que corresponden respectivamente a una distribución exactamente igualitaria y una distribución de máxima segregación. El valor de estos índices se puede

Publicado en formato digital: Prof. Aníbal Marcelo Mignone. UN ANÁLISIS DE LA SEGREGACIÓN SOCIOESPACIAL EN EL GRAN RESISTENCIA. Revista Geográfica Digital. IGUNNE. Facultad de Humanidades. UNNE. Año 8. No 16. Julio - Diciembre 2011. ISSN 1668-5180 Resistencia, Chaco.

En: http://hum.unne.edu.ar/revistas/geoweb/default.htm 
interpretar como la proporción del grupo minoritario que tendría que cambiar de residencia o ser relocalizado para obtener una distribución igualitaria en las unidades territoriales (Massey y Denton, 1998). La diferencia radica en que el IS compara la diferencia entre la proporción de individuos del grupo minoritario y la proporción del resto de población, mientras que el ID compara proporciones de dos grupos y no del grupo respecto al resto.

El cuadro № 1 , revela que los menores valores de segregación para los índices calculados, corresponden a variables vinculadas con situaciones de precariedad educativa o laboral5. Por ejemplo, si el resultado es 0,121 , esto significa que se debe relocalizar un $12 \%$ de la población ocupada que no realiza aportes provisionales, para que no exista segregación residencial. Los índices inferiores al $25 \%$ muestran que, por radio censal, no existe una zona de la ciudad que agrupe la mayor parte de la población ocupada sin aportes previsionales, algo similar a lo que ocurre en el conjunto de los ocupados con baja calificación laboral y los jefes de hogar con primaria incompleta, los que aparentemente no están restringidos a un único espacio, aunque es más factible de asociarlos con la periferia de la misma.

La población analfabeta o la que no tiene obra social, manifiestan una concentración mediabaja, en los radios censales del aglomerado; aunque del conjunto de las variables de carencias socioeconómicas, es mayor la segregación en el conjunto de jóvenes con abandono escolar, que (como se verá posteriormente) se congregan en la periferia norte y sur del Gran Resistencia.

En cambio, las variables que indican una mejor situación educativa o socioeconómica, reflejan una segregación media y media-alta. Esta sectorización de la población, se asocia con la ya mencionada cuestión de dominación y prestigio, porque las personas buscan los mejores lugares para residir y con individuos de la misma condición social. De no poder hacerlo en el área central, se instalan en zonas residenciales (generalmente barrios de una sola planta) que cuentan con todos los servicios básicos y buenas vías de circulación hacia el sector central. Estos lugares, como veremos más adelante, aparecen relacionados con el "cono de alta renta" que se prolonga por el noreste de Resistencia, entre las avenidas Sarmiento e Italia.

\section{Cuadro $N^{\circ} 1$}

Índices de segregación por radio censal. Gran Resistencia, 2001

\begin{tabular}{|c|c|c|c|}
\hline \multicolumn{2}{|c|}{ Índice de Segregación (IS) } & \multicolumn{2}{c|}{ Índice de Disimilitud (ID) } \\
\hline No aporta & 0,121 & No aporta & 0,153 \\
\hline Baja calificación & 0,140 & Baja calificación & 0,181 \\
\hline Jefe Primaria & 0,212 & Jefe Primaria & 0,234 \\
\hline Analfabetos & 0,303 & Sin obra social & 0,316 \\
\hline Sin obra social & 0,316 & Analfabetos & 0,333 \\
\hline Establecimiento Privado & 0,390 & Abandono escolar & 0,360 \\
\hline Abandono escolar & 0,419 & Establecimiento Privado & 0,376 \\
\hline Jefe Superior & 0,465 & Jefe Superior & 0,410 \\
\hline Patrones & 0,555 & Patrones & 0,502 \\
\hline
\end{tabular}

Fuente: Elaboración propia según base de datos INDEC 2001.

\section{5- LA CONCENTRACIÓN DE LA POBLACIÓN}

Los indicadores de concentración permiten conocer acerca de la ocupación de un espacio físico (superficie) por parte de un grupo de población en el medio urbano. En este caso, cuanto menor sea la porción de espacio urbano ocupado por un grupo, más concentrado y segregado se encuentra. Por lo tanto, es una medida que considera la densidad relativa del grupo en las unidades espaciales en relación a su densidad media en el conjunto urbano (Massey 1985: 289).

Para medir la concentración, se utilizó el índice Delta (Duncan, 1961 en Martori y Hoberg, 2004) que calcula la diferencia entre la proporción de la población de un grupo en cada unidad respecto al total del grupo en la ciudad y la proporción de la superficie de cada unidad con el total de la ciudad. También se puede interpretar como el porcentaje de población del grupo $\mathrm{X}$ que ha de cambiar de residencia para obtener una distribución uniforme en toda la ciudad.

Los resultados revelan nuevamente una menor concentración de población por radio censal en las variables negativas para el desarrollo profesional, laboral y económico de las habitantes y una mayor segregación en el conjunto con mejor condición socioeconómica (cuadro № 2). Al respecto, se 
Revista Geográfica Digital. IGUNNE. Facultad de Humanidades. UNNE. Año 8. № 16. Julio - Diciembre 2011. ISSN 1668-5180 Resistencia, Chaco

debe considerar la incidencia en el tamaño de los radios censales, ya que en la periferia de la ciudad se localiza la población con menores recursos pero la superficie de los radios crecen. En cambio, los que cuentan con una condición económica privilegiada, se ubican (como se verá en el siguiente apartado) en el sector central de la ciudad, dónde el área de cada unidad territorial se reduce6.

\section{Cuadro $\mathrm{N} 2$}

Índice de concentración Delta de Duncan e Índice de Autocorrelación de Moran. Gran Resistencia, 2001

\begin{tabular}{|c|c|c|c|}
\hline \multicolumn{2}{|c|}{ Delta de Duncan } & \multicolumn{2}{c|}{ Índice de Moran } \\
\hline Analfabetos & 0,532 & Sin remuneración & 0,006 \\
\hline Sin obra social & 0,527 & Abandono escolar & 0,273 \\
\hline Jefe Primaria & 0,536 & No calificada & 0,287 \\
\hline No calificada & 0,560 & Analfabetos & 0,352 \\
\hline Abandono escolar & 0,561 & Sin obra social & 0,364 \\
\hline Sin remuneración & 0,589 & Establecimiento. Privado & 0,476 \\
\hline Establecimiento Privado & 0,695 & Jefe Primaria & 0,522 \\
\hline Jefe Superior & 0,718 & Patrones & 0,569 \\
\hline Patrones & 0,761 & Jefe Superior & 0,718 \\
\hline
\end{tabular}

Fuente: Elaboración propia según base de datos INDEC 2001.

No obstante, el índice de concentración para todas las variables supera el 50\%, por lo tanto la segregación es alta, aunque más acentuada en los indicadores vinculados a una mejor condición educativa o socioeconómica; ya que habría que mover el 70\% de esta población para que no exista segregación, porque la proporción de cada grupo en los radios censales con respecto al total del grupo en la ciudad es elevada. Esto está indicando condiciones de fuerte segregación o "hipersegregación" porque como dice Massey (1985), los valores superan el 60\% de concentración en la ciudad.

De otra manera, los bajos salarios y la condición de informalidad de los perceptores de ingresos en las familias carenciadas, obliga a los restantes integrantes que residen en estos hogares a ingresar al mercado laboral en forma temprana, por lo que es de esperar que abandonarán tempranamente la escuela o serán propensos al rezago escolar (González et. al., 2007). Muchos de ellos, probablemente pasarán a conformar los barrios de pobreza, con gran deterioro urbano, cada vez más concentrados en el espacio urbano y de no mediar una acción por parte del estado que permita mejorar la precariedad laboral de sus habitantes seguirá aumentando las condiciones de pobreza estructural, fragmentando aún más el espacio urbano en el Gran Resistencia.

\section{6- LA AUTOCORRELACIÓN ESPACIAL EN EL GRAN RESISTENCIA}

La autocorrelación espacial permite medir la correlación de una variable consigo misma y considera los atributos vinculados con aspectos espaciales. Para medir la autocorrelación, se ha utilizado el Índice de Morán (I), en el cuál los valores van de -1 a 1 . Un índice de 1 indica una autocorrelación espacial positiva, un valor 0 indica un patrón aleatorio y -1 indica una fuerte autocorrelación espacial negativa7. El índice de Morán permite vincular en forma conjunta la dependencia entre lugares próximos y valores de variables o atributos que nos interesan (Velázquez y Cepeda, 2004).

Los valores muestran una autocorrelación espacial positiva para todas las variables estudiadas (cuadro $\mathrm{N}^{\circ} 2$ ), existiendo una contigüidad espacial en las unidades censales que, de acuerdo al valor de Moran, permitiría suponer la conformación de "enclaves". Esta situación, es más visible en el conjunto de población que tiene una aventajada situación socioeconómica, ya que existe una mayor autocorrelación en los jefes de hogar con estudios superiores y en el grupo de patrones con calificación profesional. Esto es importante, porque el aglomerado Gran Resistencia se caracteriza por un alto índice de pobreza, entonces los "enclaves de riqueza" adquieren una mayor visibilidad en los indicadores de segregación socioespacial.

Publicado en formato digital: Prof. Aníbal Marcelo Mignone. UN ANÁLISIS DE LA SEGREGACIÓN SOCIOESPACIAL EN EL GRAN RESISTENCIA. Revista Geográfica Digital. IGUNNE. Facultad de Humanidades. UNNE. Año 8. № 16. Julio - Diciembre 2011. ISSN 1668-5180 Resistencia, Chaco.

En: http://hum.unne.edu.ar/revistas/geoweb/default.htm 
De manera opuesta, las restantes variables asociadas con las carencias de la población cuentan con autocorrelaciones bajas y medias; no obstante, el índice de Moran es destacable en el indicador de jefes de hogar con primaria incompleta.

Con el I de Morán se obtiene un indicador global de autocorrelación de las variables analizadas, aunque el grado de relación significativo entre estas y las unidades censales ocurre sólo en determinadas zonas de la ciudad. Para esta cuestión, se recurre al $I_{i}$ de Moran (Anselin, 1995), indicador local de autocorrelación espacial (LISA) que tienen como objetivo que el estadístico obtenido indique la presencia de subáreas de dependencia espacial dentro del espacio general dado8.

Con la utilización del software Geoda, se obtienen los clusters con presencia alta de miembros de un grupo, pudiendo identificar radios censales con alta manifestación de un grupo rodeado de radios con presencia también alta de población en la misma situación (condición HighHigh), o bien unidades con presencia alta rodeadas de espacios con presencia baja (circunstancia High-Low). Con el mapa de Cluster Server de Geoda se observan los lugares estadísticamente significativos según la correlación espacial: con rojo para la asociación alto-alto, con azul para bajobajo, el celeste muestra el vínculo bajo-alto y el color rosado corresponde alto-bajo. En cambio, los que están en blanco corresponden a valores con nula significancia.

Se han agrupado los planos según el valor del Moran global y atendiendo especialmente a la distribución espacial de cada variable en la cartografía urbana. Los planos $\mathrm{N}^{\circ} 1$ y $\mathrm{N}^{\circ} 2$, vinculados con el trabajo informal y las personas ocupadas en empleos considerados no calificados, ya comienzan a revelar espacios sectorizados dentro de la ciudad, destacándose el sector noreste, en coincidencia con los barrios de Villa Prosperidad, Barrio Vargas I y II, entre otros; y por el suroeste con Villa Marín, Villa El Dorado, Juan B. Alberdi, Barrio Nazareth, Villa Aeropuerto.

Los planos $\mathrm{N}^{\circ} 3, \mathrm{~N}^{\circ} 4$ y $\mathrm{N}^{\circ} 5$ han sido agrupados considerando el incremento del número de radios que tienen una autocorrelación con valores alto-alto en las variables de carencias. Como se observa en la cartografía, se destaca el tercer anillo que integra la periferia externa del aglomerado, dónde la convergencia de situaciones en un mismo espacio puede primordialmente estar sujeta a cuestiones socioeconómicas forzadas, como la falta de empleo o los ingresos bajos (Sabatini, 2003). En este sentido, lo que estaría indicando esta segregación socio-espacial impuesta por la condición socioeconómica, es la falta de cobertura social de la población, la precariedad laboral, el bajo nivel educativo de los jefes de hogar y la pobreza estructural de estos conjuntos.

Asimismo, el tercer anillo envolvente de la ciudad, está compuesto mayormente por asentamientos informales mediante la ocupación ilegal de los terrenos. Son áreas con dificultades en el acceso a infraestructura básica (cuentan con servicio de agua con canilla pública y servicio de luz "enganchada") y dónde se observa que va disminuyendo la accesibilidad y se produce el pasaje a lugares dónde el precio del suelo es muy inferior, coincidente con lugares dónde faltan los servicios básicos, aumenta el riesgo de inundaciones y de contaminación ambiental9. También se puede relacionar con el tipo de calificación laboral de las personas, en las cuáles se entremezclan los ocupados no calificados con aquellos que son trabajadores informales (cf. Meichtry y Mignone, 2003).

Plano $\mathrm{N}^{0} 1$

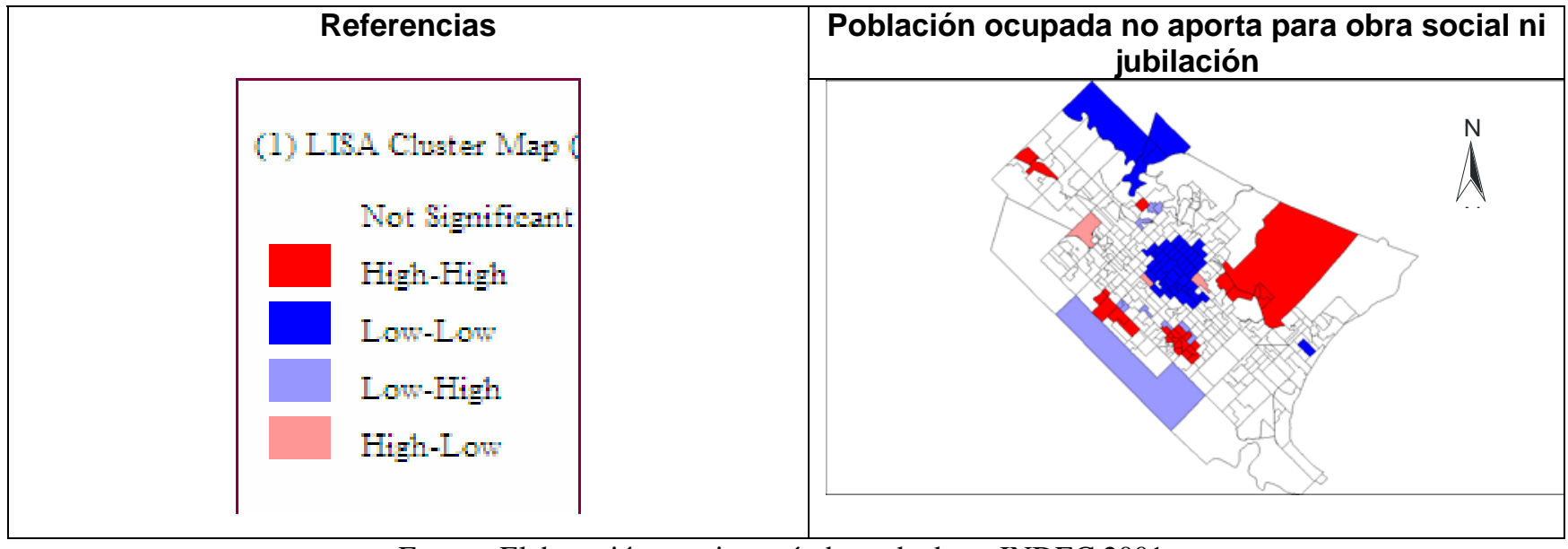

Fuente: Elaboración propia según base de datos INDEC 2001.

Publicado en formato digital: Prof. Aníbal Marcelo Mignone. UN ANÁLISIS DE LA SEGREGACIÓN SOCIOESPACIAL EN EL GRAN RESISTENCIA. Revista Geográfica Digital. IGUNNE. Facultad de Humanidades. UNNE. Año 8. № 16. Julio - Diciembre 2011. ISSN 1668-5180 Resistencia, Chaco.

En: http://hum.unne.edu.ar/revistas/geoweb/default.htm 


\begin{tabular}{|c|c|c|}
\hline Población ocupada no calificada & Abandono escolar \\
\hline & & \\
\hline
\end{tabular}

Fuente: Elaboración propia según base de datos INDEC 2001.

En cierta medida, estos barrios de invasión son colindantes con población residente en los barrios planificados. Por otra parte, los planos presentados hasta el momento, indican que los valores bajo-bajos coinciden con el sector central del aglomerado, y se prolongan por las avenidas 9 de Julio y 25 de Mayo.

Por otra parte, está comprobado empíricamente que cuánto mayor es el nivel de instrucción formal de la población, mayor es su posibilidad de acceso a puestos con alta calificación laboral y por lo tanto, mejor remunerados. En este caso, los patrones con calificación profesional y los jefes de hogar con estudios superiores, aparecen en el sector central de la ciudad y se extienden hacia el noreste según los valores alto-alto de la autocorrelación (Plano $N^{\circ} 8, N^{0} 9$ y $\left.N^{0} 10\right)$. En el caso del conjunto de patrones, al ser los propietarios de medianas o grandes empresas, cuentan con buenos ingresos, que les permite poder "elegir" el lugar de residencia dentro del espacio urbano.

Población Analfabeta

Fuente: Elaboración propia según base de datos INDEC 2001. 


\section{Población sin obra social}

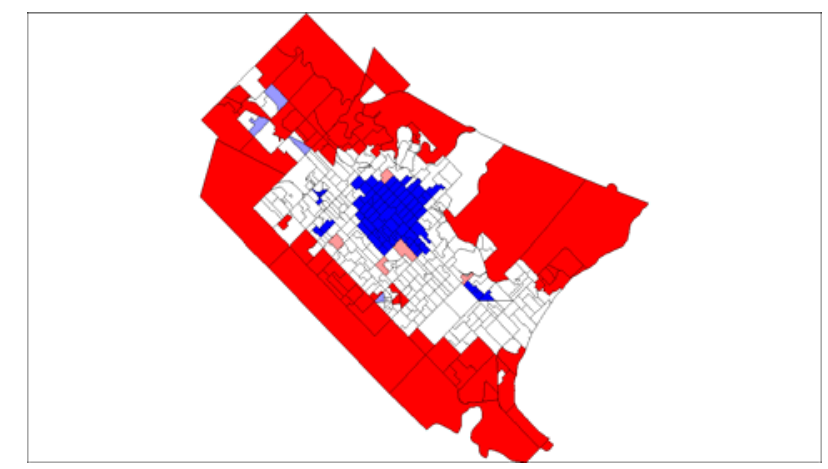

Plano $\mathrm{N}^{\circ} 7$

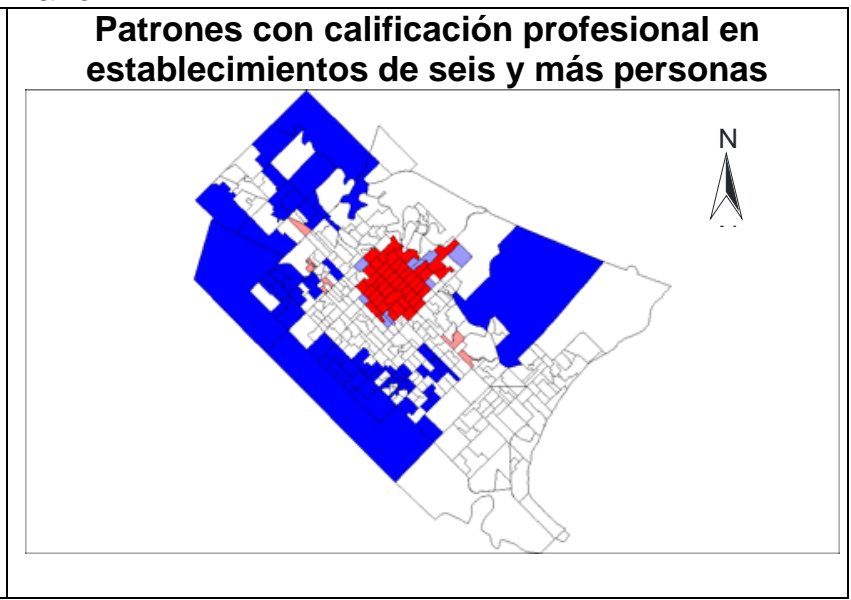

Fuente: Elaboración propia según base de datos INDEC 2001.
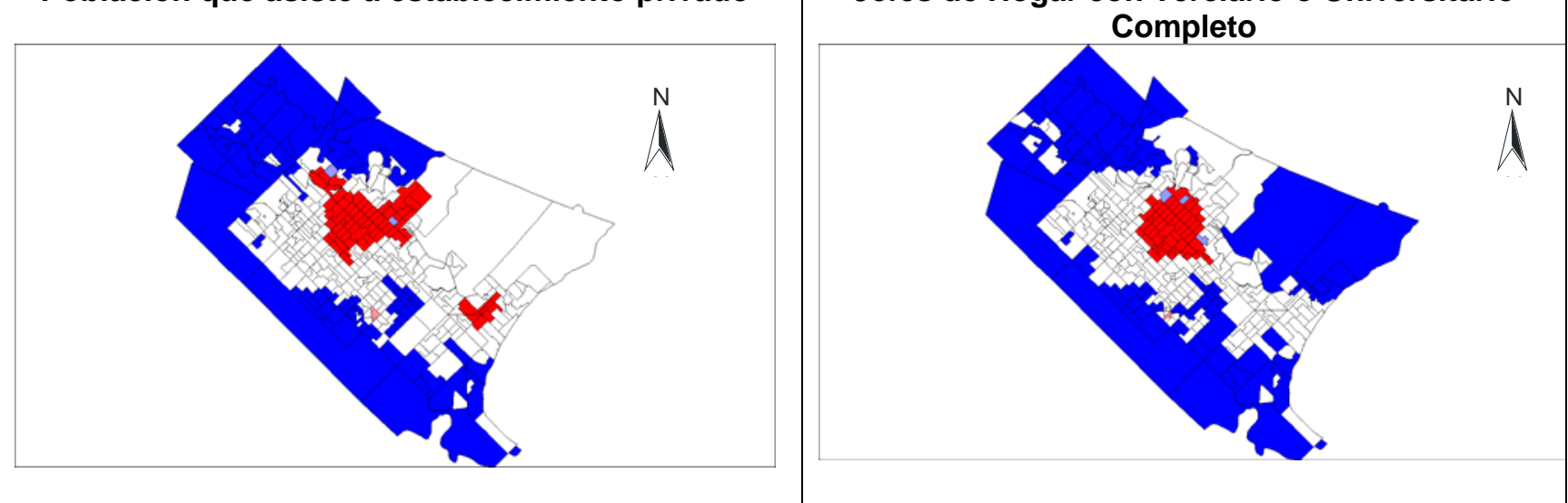

Fuente: Elaboración propia según base de datos INDEC 2001.

En cuanto a la población que asiste a establecimiento privado, indirectamente está reflejando las áreas que tienen hogares con un mayor poder adquisitivo. Los radios con mayores porcentajes nuevamente están en el sector central de la ciudad, con un eje de prolongación hacia el noreste, enmarcado entre las avenidas Sarmiento e Italia y prolongándose por los barrios "Los Troncos", "Los Teros" y "Judicial" y extendiéndose en dirección a la ruta nacional $N^{\circ} 16$, con viviendas residenciales de alta categoría. Más aún, se puede estimar que esta condición se va a reforzar con la autovía de la ruta nacional $N^{\circ} 16$, ya que en la actualidad se han construido nuevos barrios cerrados, en las proximidades de Villa Fabiana. Estos terrenos fueron ocupados a partir de la década de 1990; originariamente eran terrenos suburbanos, con vegetación natural y alto riesgo de inundaciones por la cercanía del río Negro. Cuando se construyeron las defensas, se lotearon los terrenos y se crearon barrios para (entre otros) profesionales de la salud, juristas y empresarios. Hacia esta dirección se está extendiendo el "cono de alta renta o de riqueza", con sectores residenciales de clase media-alta y alta.

De manera opuesta, los valores bajo-bajo (color azul) en toda la periferia urbana, nos exime de mayores comentarios. 
Revista Geográfica Digital. IGUNNE. Facultad de Humanidades. UNNE. Año 8. № 16. Julio - Diciembre 2011. ISSN 1668-5180 Resistencia, Chaco

\section{7- CONCLUSIONES}

Los resultados obtenidos a nivel de radio censal, permiten reconocer una fragmentación socioespacial en el interior de la ciudad. Si se comparan las dimensiones de la segregación socioespacial, de acuerdo con las variables analizadas, se observa que la segregación aumenta en aquello indicadores vinculados con una mejor condición socioeconómica de la población y tiende a ser menor en los datos que responden a situaciones de carencias socioeconómicas.

El conjunto de variables vinculadas con el déficit educativo formal, la falta de cobertura social y la población que trabaja en empleos considerados como no calificados, muestran valores de segregación bajos o medio-bajos, aunque al calcular el índice de concentración adquiere un valor medio, al igual que al calcular la autocorrelación de Moran. No obstante, al observar la autocorrelación local, habría un mayor aporte de las unidades censales localizadas por el nortenoreste y en los sectores sur-suroeste.

La segregación sería mayor, al observar los datos de las variables que indican una mejor situación socioeconómica y educativa de los habitantes, ya que en todas las dimensiones evaluadas, los valores tienden a ser altos. La localización espacial, observando la distribución de la autocorrelación local, coincide con el área central y se extiende hacia en noreste.

El patrón de segregación que se podría reconocer en el Gran Resistencia posee tres características: existe una concentración de la población con mejor condición socioeconómica, que puede elegir y acceder a los lugares donde la renta del suelo es elevada. Este grupo conforma el "cono de alta renta" que parte del casco central y se prolonga con sus vértices guiado por los ejes viales de las avenidas Sarmiento y San Martín.

En segunda instancia: se detecta la presencia de áreas dónde entremezclan los caracteres analizados conformando un segundo anillo territorial. En tercer lugar, aparece lo que puede considerar una segregación socioespacial que es impuesta por las condiciones socioeconómicas, que concentra la población con mayores carencias sociales y económicas, en unidades censales localizadas en la periferia externa de la ciudad.

Es decir que la distribución opera de acuerdo a una lógica espacial, dónde la población con mayor nivel educativo y mejor condición laboral aparece en el área central, y de allí va disminuyendo anularmente hasta llegar a la periferia dónde los habitantes tienen mayor precariedad laboral y ciclo básico incompleto.

\section{8- NOTAS}

1- El aglomerado Gran Resistencia, está integrado por cuatro municipios: Resistencia (que es la capital provincial), Barranqueras (segundo municipio de importancia), Puerto Vilelas y Fontana (ambas con un fuerte pasado industrial).

2- Aglomeración de tamaño intermedio menor o ATIs menor según el número de habitantes (Vapñarsky y Gorojovsky, 1990)

3- En los últimos 50 años, el área urbana de Resistencia se expandió en forma muy acelerada. El declive experimentado por la crisis del algodón generó el incremento de las actividades terciarias en la capital provincial. Esto trajo como consecuencia el desplazamiento de población rural atraída por las condiciones propicias de la ciudad, ante la crisis del campo (cf. Bolsi y Bruniard, 1975)

4- Encuestas realizadas a los habitantes de asentamientos informales en el año 2001 y 2002, permitieron conocer que unas de las causas por el cuál optaron por localizarse en un asentamiento informal, era ante la imposibilidad de seguir conviviendo con los padres en viviendas con espacios muy reducidos y por la necesidad de conformar su propia familia y contar con su propia vivienda (Meichtry y Mignone, 2003; Mignone, 2005).

5- La interpretación de los resultados requiere no solo el conocimiento de lo que se mide, también hay que considerar el ámbito y la realidad concreta que se pretende medir, ya que formalmente no existe una escala de interpretación preestablecida universalmente que permita definir a partir de qué valores un índice de segregación reconoce condiciones de desigualdad territorial (cf. Lavía Martínez, 2008). La lectura acerca de los trabajos científicos previos referentes a la segregación residencial, permiten considerar para esta instancia, tres parámetros de medición: cuando el índice es menor a 25\%, hablamos de baja segregación, entre $25 \%$ y $50 \%$ es media y más de $50 \%$ se puede considerar alta. 6- Se recuerda que la división espacial de los radios censales, esta en relación con la cantidad de viviendas. En el centro de la ciudad, dónde el número de viviendas es mayor, el radio abarca una 
superficie menor; en cambio, las moradas en la periferia urbana se encuentran más distanciadas entre sí, por ello el área del radio se acrecienta considerablemente.

7- La matriz de pesos especiales construida con el software Geoda 0.9.5, fue la "matriz de contactos binarios", atendiendo a un criterio de contigüidad de tipo Queen o Reina, con el cuál se concibe que serán vecinas de i las unidades censales que comparten algún lado o vértice con i.

8- El análisis espacial se realiza a través del reconocimiento de patrones locales de identificación espacial. Este método descompone el índice I de Moran y verifica en cuánto contribuye cada unidad espacial a la formación del valor general, permitiendo obtener un valor de significatividad para cada cluster formado por los valores similares de cada unidad espacial y sus vecinos

9- Se recuerda que la periferia norte-noreste esta atravesada por el río Negro y el conjunto de lagunas que restringen la ocupación por el noreste. Por otra parte, muchos de los barrios informales localizados en el sur de la ciudad, se encuentran cercanos a lagunas de oxidación.

\section{9- BIBLIOGRAFÍA}

Anselin, Luc. 1995. "Local indicators of spatial association-LISA". En: Geographical Análisis. Volumen No 27: 93-115.

Arriagada Luco, Camilo y Jorge Rodríguez Vignoli. 2003. "Segregación residencial en áreas metropolitanas de América Latina: magnitud, características, evolución e implicaciones de política". En: Serie Población y Desarrollo $\mathbf{N}^{\circ}$ 47. Octubre de 2003. CELADE- UNFPA. Santiago de Chile, Chile. Disponible en Internet: http://www.eclac.cl/publicaciones/xml/7/13877/lcl1997-P01.pdf

Barreto, Miguel y Lorena Sánchez. 2000. "El crecimiento de las ciudades intermedias del noreste argentino en el contexto de las transformaciones regionales". En: Seminario El rol de las ciudades intermedias iberoamericanas. Facultad de Arquitectura y Urbanismo, UNNE. Versión en CD-Rom.

Bolsi, Alfredo y Enrique Bruniard. 1975. "El proceso histórico y los caracteres demográficos y socioeconómicos de la ciudad de Resistencia". En Folia Histórica del Nordeste $\mathbf{N}^{\circ} \mathbf{1}$. Instituto de Historia, Universidad Nacional del Nordeste, Resistencia.

Borsdorf, Axel. 2003. "Cómo modelar el desarrollo y la dinámica de la ciudad latinoamericana". Reviste Eure. Volumen 29, № 86. Santiago de Chile

Buzai, Gustavo. 2003. Mapas sociales urbanos. Lugar Editorial, Buenos Aires.

Castells, Manuel. 1974. La cuestión urbana. Editorial Siglo XXI, Buenos Aires.

Duncan, O. y Duncan, B. 1955. "A methodological analysis of segregation indexes". En American Sociological Review. Volumen 20. № 2:210-217.

Formiga, Nidia. 2000. La diferenciación socioespacial y los espacios subjetivos de los bahienses. Relación global-local en la estructuración del espacio urbano. Tesis doctoral. Facultad de Geografía e Historia. Universidad Complutense de Madrid, Madrid. Septiembre de 2000. Disponible en Internet: http://eprints.ucm.es/tesis/19972000/H/0/H0041801.pdf.

Foschiatti de Dell' Orto, Ana María. La población del Chaco en los últimos 30 años. Diario Norte, 24 de mayo de 1992. Pp. 18.

González, Leandro, Enrique Peláez y Verónica Herrero. 2007. "Vulnerabilidad social, rezago y deserción escolar en el Gran Córdoba (Argentina) 2001". En: IX Jornadas de Estudios de Población (AEPA). Huerta Grande, Córdoba, Argentina. 31 de octubre, 1y 2 de noviembre de 2007. Versión en CD-Rom.

Janoschka, Michel. 2002: "El nuevo modelo de la ciudad latinoamericana: fragmentación y privatización". Revista Eure. Volumen 28, № 85. Santiago de Chile. Diciembre de 2002

Lavía Martínez, Cristina. 2008. La segregación residencial de la población extranjera de Bilbao. Revista ACE (Arquitectura, ciudad y entorno). Año III, Número 8, octubre de 2008. Disponible en Internet: http://www.upcommons.upc.edu/revistes/bitstream/2099/6578/.../4 LAVIA.pdf.

Martori i Cañas, Joan y Karen Hoberg. 2004. "Indicadores cuantitativos de segregación residencial. El caso de la población inmigrante en Barcelona". En: Scripta Nova. Vol. VIII, No 169. Universidad de Barcelona, Barcelona. 15 de julio de 2004.

Massey, Douglas y Nancy Denton. 1998. "The dimensions of residential segregation". En: Social Forces. Volumen 67, № 2: 281-315.

Massey, Douglas. 1985."Ethnic residential segregation: a theoretical synthesis and empirical review". En: Sociology and Social Research $\mathbf{N}^{\circ}$ 69. Pp. 315-350.

Publicado en formato digital: Prof. Aníbal Marcelo Mignone. UN ANÁLISIS DE LA SEGREGACIÓN SOCIOESPACIAL EN EL GRAN RESISTENCIA. Revista Geográfica Digital. IGUNNE. Facultad de Humanidades. UNNE. Año 8. № 16. Julio - Diciembre 2011. ISSN 1668-5180 Resistencia, Chaco.

En: http://hum.unne.edu.ar/revistas/geoweb/default.htm 
Meichtry, Norma y Aníbal Mignone. 2003. Movilidad territorial. Una contribución a partir de la población en asentamientos periféricos espontáneos en Resistencia, Chaco, en la década de 1990. VI Jornadas de la Asociación de Estudios de Población de la Argentina. Asociación de Estudios de Población de la Argentina (AEPA) y la Universidad Nacional del Comahue, Neuquén. 14 al 16 de noviembre de 2001. Pp. 324-339.

Mignone, Aníbal. 2001. Asentamientos espontáneos en Resistencia durante la década de 1990. Condiciones sociodemográficas. Primer Congreso Nacional sobre Problemáticas Sociales Contemporáneas. Facultad de Humanidades y Ciencias de la Universidad Nacional del Litoral, Santa Fe. Versión CD-ROM.

Mignone, Aníbal. 2005. La movilidad territorial desde la perspectiva de la población instalada en asentamientos espontáneos en el Gran Resistencia, a principios del siglo XXI. VII Jornada Argentina de Estudios de la Población (AEPA). 5 al 7 de noviembre de 2003. Tafí del Valle, Tucumán, Argentina. Pp. 315-328.

Rodríguez Vignoli Jorge. 2001. "Segregación residencial socioeconómica: ¿qué es?, ¿cómo se mide? ¿qué está pasando? ¿importa?" Serie Población y Desarrollo $\mathbf{N}^{\circ}$ 16. CELADE-UNFPA, Santiago de Chile.

Sabatini Francisco y Carlos Sierralta. 2006. Medición de la Segregación Residencial: Meandros Teóricos y Metodológicos, y Especificidad Latinoamericana. Documento de Trabajo $\mathrm{N}^{\circ}$ 38. Instituto de Estudios Urbanos_Facultad de Arquitectura, Diseño y Estudios Urbanos de la Pontificia Universidad Católica de Chile. Disponible en Internet: http://www.ieut.cl/media/archivos/sabatinisierralta dt38.pdf.

Sabatini, Francisco. 2000. "Reforma de los mercados de suelo en Santiago, Chile: efectos sobre los precios de la tierra y la segregación residencial". En Revista EURE. Volumen XXVI, № 77, Pp. 49-80. Disponible en Internet: http://www.scielo.cl/scielo.php?script=sci arttext\&pid=S025071612000007700003\&lng=es\&nrm=iso

Sabatini, Francisco. 2003. La segregación social del espacio en las ciudades de América Latina. Documentos del Instituto de Estudios Urbanos y Territoriales, Serie Azul № 35 . Pontificia Universidad Católica de Chile. Santiago de Chile. Disponible en Internet: http://www.territorioysuelo.org.

Valenzuela de Mari. Cristina. 2000. "Modalidades de expansión en ciudades intermedias argentinas. Urbanización planificada y espontánea en el Gran Resistencia. 1970-1998". Revista de Geografía Norte Grande, No 26. Instituto de Geografía de la Pontificia Universidad Católica de Chile. Santiago de Chile, Chile.

Vapñarsky, César A. y Gorojovsky, Néstor. 1990. El crecimiento urbano en la Argentina. Instituto Internacional del Medio Ambiente y Desarrollo. Buenos Aires. Grupo Editor Latinoamericano.

Velázquez, Guillermo y Rosana Cepeda. 2004. "Análisis de asociación espacial en variables de calidad de vida en la Argentina". En: Revista Geográfica. Instituto Panamericano de Geografía e Historia. № 136, Julio- diciembre de 2004. Pp. 109-132. 